\title{
A Note on the Existence of Nash Equilibrium in Games with Discontinuous Payoffs
}

\author{
J. Rupert J. Gatti
}

February 2005

CWPE 0510

Not to be quoted without permission 


\begin{abstract}
:
This paper generalises the approach taken by Dasgupta \& Maskin (1986) and Simon (1989) and provides necessary and sufficient conditions for the existence of pure and mixed strategy Nash equilibrium in games with continuous strategy spaces and discontinuous payoff functions. The conditions can be applied widely, and examples for existence of pure strategy and monotonic equilibria in First-Price auctions are provided. The conditions are also appropriate for ensuring that computer generated equilibrium solutions can be extended to continuous strategy spaces.
\end{abstract}

Keywords: Nash Equilibrium, Discontinuous Payoff Function, FirstPrice Auctions.

JEL Classification: C70, D44 


\title{
A Note on the Existence of Nash Equilibrium in Games with Discontinuous Payoffs
}

\author{
J. Rupert J. Gatti ${ }^{1}$
}

The seminal paper on the existence of Nash equilibria in games with discontinuous payoff functions is Dasgupta \& Maskin (1986). In that paper Dasgupta \& Maskin weaken the assumption of continuous payoff functions made in the classical existence theorems (e.g. Debreu (1952)) and generate existence results for mixed strategy Nash equilibrium in a family of games possessing a very specific form of discontinuity in their payoffs. Simon (1987) and Reny (1999) generalize their results to a broader set of games, while Reny allows for the analysis of pure strategy, as well as mixed strategy, Nash equilibria. This paper strictly generalizes the results of Dasgupta \& Maskin and Simon, but in a different way to Reny, allowing existence of pure and mixed strategy Nash Equilibrium to be proven in classes of games not considered by Reny. The usefulness of this result is demonstrated with an application to First-Price auctions which itself extends existence theorems provided by Maskin \& Riley (2000), Athey (2001), Jackson et al. (2001) and Reny \& Zamir (2004).

The general approach taken by Dasgupta \& Maskin (1986) to the problem of determining conditions for the existence of a mixed strategy Nash equilibrium in games with compact strategy spaces and discontinuous payoffs is to consider a sequence of consecutively finer finite approximations of the strategy space, converging to the compact space at the limit. Mixed strategy Nash equilibria exist for games with finite strategy spaces (Nash 1951), so conditions are derived which

\footnotetext{
${ }^{1}$ Faculty of Economics, University of Cambridge. email: rupert.gatti@econ.cam.ac.uk
} 
ensure that the limit of a sequence of 'finite game' Nash equilibria is itself a Nash equilibrium of the limit game. As the limit of any sequence of 'finite game' Nash equilibria will be non-empty, and there exist games without Nash equilibria (Sion \& Wolfe (1957)), it must be the case that additional restrictions on the game are required.

Dasgupta \& Maskin consider games where the points of discontinuities in each player's payoff function can be represented by a finite number of continuous and one-to-one functions of the strategies of other players. The main result of their paper (1986a, Theorem 5, p.14) identifies additional conditions on the agents' payoff functions which ensure that the limit of any sequence of 'finite game' Nash equilibria is a Nash equilibrium of the limit game.

Simon explains that these conditions are stronger than is necessary. If a particular sequence of finite approximations of the strategy space is referred to as a 'finite test sequence', then for any finite test sequence we may consider a 'limit set' of mixed strategies which are the limit of a sequence of (mixed strategy) Nash equilibria in games along the test sequence. The conditions identified by Dasgupta \& Maskin effectively ensure that every element of every limit set for every finite test sequence is a Nash equilibrium of the limit game. Simon (1987, Lemma, p.575) derives conditions which ensure that every element of the limit set for a particular finite test sequence is a Nash equilibrium of the limit game. He then goes on to identify conditions on the payoff functions of agents which are sufficient to satisfy the conditions in his lemma . The increased generality of these results is that the conditions need not hold true for every test sequence, but only for one.

Reny (1999) takes a different approach to that of Dasgupta \& Maskin and Simon, considering a sequence of approximations of the payoff function rather than of the strategy space. He obtains conditions for the existence of Nash equilibrium in the limit game if Nash exist for 
each approximation game, and shows that these conditions are strictly more general than those obtained by Simon. Moreover Reny's results are applicable to pure as well as mixed strategy games.

However, even conceptually, it is clear that Simon's conditions are stronger than required for existence. Considering just one finite test sequence, it is not necessary that every element of the limit set be a Nash equilibrium of the limit game but just that there exists an element where this is true. Furthermore we need not restrict the test sequence to include only finite strategy spaces. The main result in this paper (Theorem 1) provides sufficient conditions for an element of a limit set to be a Nash equilibrium of the limit game, and does not restrict the test sequence to be finite. Furthermore it is shown that an acceptable test sequence satisfying the conditions exists for any Nash equilibrium of the limit game.

The characteristic feature of Theorem 1 is that it requires the identification of a specific test sequence and a specific element of the limit set. Thus, for example, it is particularly applicable for testing the generality of equilibria obtained by computer simulation (with necessarily finite strategy spaces) to games with continuous strategy spaces. For theoretical work however, where conditions on the primitives of the model are probably more applicable, this requirement may appear overly restrictive. However Theorem 1 can be applied in tandem with any primitives based existence theorem to extend the existence result to a wider class of games. Theorem 1, in combination with any condition sufficient for the creation of an acceptable test sequence, will ensure the existence of equilibria in the limit game and existence results generated using alternative approaches, see for example Baye et al (1993) and Tian (1992), can be applied if desired. This feature is highlighted in the application.

In the following section the primary notation and definitions are 
introduced and the main result of the paper stated. An application of this result to First-Price auctions is provided in Section 2, while Section 3 concludes.

\section{The Existence Result.}

Consider a game $G=(S, U)$, where $S=\mathrm{X}_{i \in I} S_{i}, I$ is the finite set of all agents, $S_{i}$ is a compact metric space of strategies for agent $i \in I$ , $U=\mathrm{X}_{i \in I} U_{i}$ and $U_{i}: S \rightarrow \mathbf{R}$ is the utility function for agent $i$. I assume that the utility function is bounded and Borel measurable. Let $N(G)$ denote the set of (pure strategy) Nash Equilibria of the game $G$, we are interested in obtaining conditions that will ensure that $N(G)$ is non-empty. To do this we consider a sequence of games all possessing Nash equilibria and converging to the game $G$.

Let $F\left(S_{i}\right)$ be the set of closed, non-empty subsets of $S_{i}$ and $F(S)=$ $\mathrm{X}_{i \in I} F\left(S_{i}\right)$. Paired with the Hausdorff metric, $d^{H}, F(S)$ is a compact metric space (Hildenbrand 1974, p17). ${ }^{2}$

Define a Test Sequence for $S, \tau: \mathbf{N} \rightarrow F(S)$, to be a mapping from the Natural numbers into $F(S)$, so $\tau(n) \subset S$, and $\lim _{n \longrightarrow \infty} d^{H}(\tau(n), S)=$ 0 . We may consider a test sequence for $S$ as a sequence of progressively finer approximations of $S$, converging to the compact metric space $S$ in the limit, but notice that the test sequence need not be finite. A test sequence, combined with the utility function $U$, can be used to generate a sequence of games $G^{n}=(\tau(n), U)$ which converges to the limit game $G$, and a corresponding sequence $N^{n} \in F(\tau(n)) \cup \varnothing$ where $N^{n}=N\left(G^{n}\right)$ is the set of all (pure strategy) Nash Equilibria of the

\footnotetext{
2 "For every two non-empty subsets $E$ and $F$ of the metric space $(X, d)$ one defines the Hausdorff distance $d^{H}(E, F)$ (with respect to the metric $d$ on $X$ ) by $d^{H}(E, F)=\inf \left\{\varepsilon \in(0, \infty) \mid E \subset B_{\varepsilon}(F)\right.$ and $\left.F \subset B_{\varepsilon}(E)\right\}$,

where $B_{\varepsilon}(E)$ denotes the $\varepsilon$-neighbourhood of $E$." (Hildenbrand, p.16)
} 
Game $G^{n}$.

Assigning the topology of weak convergence, every (non-empty) sequence in compact metric space $S$ possesses a convergent subsequence (Hidenbrand, p49). Without loss of generality we consider only convergent sub-sequences, and will call a test sequence $\tau$ acceptable when, for all $n, N^{n}$ is non-empty and $\lim _{n \longrightarrow \infty} N^{n}=N_{\tau}^{*} \in F(S)$. The existence of such a sequence is not guaranteed, but Theorem 1 identifies conditions which ensure that there exists an element $s^{*} \in N_{\tau}^{*}$ such that $s^{*} \in N(G)$ when an acceptable sequence has been identified. In fact Theorem 1 provides necessary and sufficient conditions for the existence of Nash equilibrium, thus we also know that for any Nash equilibrium of the limit game there exists at least one acceptable sequence satisfying the conditions.

Before stating Theorem 1 two further definitions are required, both of which are direct extensions of concepts developed by Simon and Reny.

Consider a test sequence $\tau$ and a convergent sequence of strategies $\left(s^{n}\right)_{n \in \mathbf{N}}$ where $s^{n} \in \tau(n), \lim _{n \rightarrow \infty} s^{n}=s^{*} \in S$ and $\lim _{n \rightarrow \infty} U_{i}\left(s^{n}\right)$ is defined for all agents $i \in I .^{3}$

Definition 1 We will say that the utility function $U$ has the complementary discontinuity property (cdp) for the sequence $\left(s^{n}\right)_{n \in \mathrm{N}}$ if, for any agent $i \in I$ where $\lim _{n \rightarrow \infty} U_{i}\left(s^{n}\right)>U_{i}\left(s^{*}\right)$ there exists another agent $j \in I$ where $\lim _{n \rightarrow \infty} U_{j}\left(s^{n}\right)<U_{i}\left(s^{*}\right)$.

The complementary discontinuity property ensures that, if an agents expected payoff decreases discontinuously along the sequence $\left(s^{n}\right)_{n \in \mathbf{N}}$ at $s^{*}$ then there exists another agent who experiences a discontinuous increase in utility. Notice that, in contrast to Simon and Reny, this property does NOT need to hold for every sequence - but is defined for

\footnotetext{
${ }^{3} \mathrm{As} S$ is compact and $U_{i}$ is bounded every test sequence will posses a sub-sequence with these properties, without loss of generality we consider only these sub-sequences.
} 
the specific sequence $\left(s^{n}\right)_{n \in \mathbf{N}} \cdot{ }^{4}$

Let $Z_{i}^{n}: \tau_{-i}(n) \rightarrow \mathbf{R}$ be the best response value function for agent $i$, where $Z_{i}^{n}\left(s_{-i}^{n}\right)=\max _{s_{i}^{n} \in \tau_{i}(n)} U_{i}\left(s_{i}^{n}, s_{-i}^{n}\right)$ and similarly let $Z_{i}\left(s_{-i}\right)=$ $\max _{s_{i} \in S_{i}} U_{i}\left(s_{i}, s_{-i}\right)$. The best response value function represents the highest level of utility agent $i$ can obtain in the game $G^{n}$ given the strategies played by all other agents. Clearly, from the definition of Nash Equilibrium, $s^{n} \in N^{n}$ if and only if, for all agents $i \in I$, $U_{i}\left(s^{n}\right)=Z_{i}^{n}\left(s_{-i}^{n}\right)$.

Definition 2 We will say that the Utility function is payoff secure (ps) for the sequence $\left(s^{n}\right)_{n \in \mathbf{N}}$ if, for all agents $i \in I$ and all $\epsilon>0$ there exists $\bar{n}<\infty$ such that for all $n \geq \bar{n}, Z_{i}^{n}\left(s_{-i}^{n}\right)>Z_{i}\left(s_{-i}^{*}\right)-\epsilon$.

Payoff security ensures that, along the sequence $\left(s^{n}\right)_{n \in \mathbf{N}}$, every agent can obtain a payoff against opponents' strategies sufficiently close to $s_{-i}^{*}$ which is almost as good as the best payoff obtainable by the agent in the limit game against $s_{-i}^{*}$. Once again, the generalization of this condition over similar conditions defined by Simon and Reny is that payoff security is defined for a specific sequence and need not hold for all possible sequences. Having introduced this terminology, the main existence theorem can now be stated.

\section{Theorem 1 Existence Theorem.}

A vector of strategies $s^{*} \in S$ will constitute a Nash equilibrium of the game $G=(S, U)$ if and only if there exists an acceptable test sequence $\tau$ and a sequence of strategies $\left(s^{n}\right)_{n \in \mathbf{N}}$, where $s^{n} \in N^{n}$ and $\lim _{n \rightarrow \infty}$ $s^{n}=s^{*}$, such that the utility function $U$

a. possesses the complementary discontinuity property for the sequence $\left(s^{n}\right)_{n \in \mathbf{N}}$ and

\footnotetext{
${ }^{4}$ Using slightly different terminology to Simon, Reny refers to this property as reciprocal upper semicontinuity rather than complementary discontinuity.
} 
b. is payoff secure for the sequence $\left(s^{n}\right)_{n \in \mathbf{N}}$.

Proof. a. Sufficiency

The proof is by contradiction.

Assume that $s^{*} \notin N(G)$, so there exists an agent $i \in I, \epsilon_{i}>0$ and $t_{i} \in S_{i}$ such that $U_{i}\left(t_{i}, s_{-i}^{*}\right)>U_{i}\left(s^{*}\right)+\epsilon_{i}$.

As the sequence is acceptable and $U$ is payoff secure along the sequence we have that

$$
\begin{aligned}
\lim _{n \rightarrow \infty} U_{i}\left(s^{n}\right) & =\lim _{n \rightarrow \infty} Z_{i}^{n}\left(s_{-i}^{n}\right) \\
& >Z_{i}\left(s_{-i}^{*}\right)-\epsilon_{i} \\
& \geq U_{i}\left(t_{i}, s_{-i}^{*}\right)-\epsilon_{i} \\
& >U_{i}\left(s^{*}\right)
\end{aligned}
$$

As $U$ possess the complementary discontinuity property along the sequence, there exists another agent $j \in I$ and $\epsilon_{j}>0$ such that

$$
\lim _{n \rightarrow \infty} U_{j}\left(s^{n}\right)<U_{j}\left(s^{*}\right)-\epsilon_{j} .
$$

Payoff security ensures that there exists $\bar{n}<\infty$ such that for all $n \geq \bar{n}$ there exists $t_{j}^{n} \in S_{i}^{n}$ where

$$
\begin{aligned}
U_{j}\left(t_{j}^{n}, s_{-j}^{n}\right) & >U_{j}\left(s^{*}\right)-\frac{\epsilon_{j}}{2} \\
& >\lim _{n \rightarrow \infty} U_{j}\left(s^{n}\right)+\frac{\epsilon_{j}}{2}
\end{aligned}
$$

Thus, for $n$ sufficiently high, $Z_{j}^{n}\left(s_{-j}^{n}\right) \geq U_{j}\left(t_{j}^{n}, s_{-j}^{n}\right)>U_{j}\left(s^{n}\right)$ and $s^{n} \notin N^{n}$, a contradiction.

b. Necessity

The proof is by construction, we show that for any $s^{*} \in N(G)$ it is possible to construct a (finite) acceptable sequence, along which the conditions hold. 
Specifically we make use of the fact that if $s^{*} \in S^{\prime} \subseteq S$ and $s^{*} \in N(G)$ then $s^{*} \in N\left(G^{\prime}\right)$ where $G^{\prime}=\left(S^{\prime}, U\right)$. Consider the finite strategy space where for all $i \in I, S_{i}^{1}=\left\{s_{i}^{*}\right\}$. Along any sequence $\tau$ such that $S_{i}^{1} \subset S_{i}^{n} \subset S_{i}^{n+1}$ such that $\lim _{n \rightarrow \infty} d^{H}\left(S^{n}, S\right)=0$ have that $s^{*} \in N\left(G^{n}\right)$ and $Z_{i}^{n}\left(s_{-i}^{*}\right)=U_{i}\left(s^{*}\right)$ along the sequence. Thus the complementary discontinuity property and payoff security are trivially satisfied along any such sequence sequence.

This completes the proof of Theorem 1.

The characteristic difference between Theorem 1 and other existence results (e.g. Reny (1999), Athey (2001)) is that Theorem 1 relies on the identification of a specific sequence of approximation games and Nash equilibria, rather than being on the primitives of the game itself. Thus it can be easily applied to equilibria generated by computer approximation to ensure they remain equilibria in continuous strategy spaces. As Athey (2001) notes, computer simulations are becoming an increasingly powerful tool for solving games with discontinuous payoffs. The result is also applicable in specific games where more restrictive 'general' properties over the primitives of the game fail. Examples of the former can be easily constructed - being any game where (for example) the 'misbehaviour' of the payoffs can be shown to occur at an irrelevant part of the payoff function.

However Theorem 1 can also be applied in less specific settings to provide existence results which are based on the primitives of the game. For example, Corollary 1 provides general conditions on the payoff function which ensure existence of a pure strategy Nash equilibrium in the game $G=(S, U)$ whenever a acceptable test sequence can be established.

Following Reny (1999), for all $s \in S$ let $U(s)=\left(U_{1}(s), \ldots U_{I}(s)\right)$. The graph of the vector payoff function is the subset of $S \times \mathbf{R}^{I}$ given by $\left\{(s, u) \in S \mathrm{xR}^{I} \mid u=U(s)\right\}$. We will say that: 
a. player $i$ can secure a payoff $\alpha \in \mathbf{R}$ at $s \in S$ if there exists an $\bar{s} \in S_{i}$ such that $U_{i}\left(\bar{s}, s_{-i}^{\prime}\right) \geq \alpha$ for all $s_{-i}^{\prime}$ in some open neighbourhood of $s_{-i}$

b. $U$ is payoff secure everywhere if for every $s \in S$ and $\varepsilon>0$ each player $i$ can secure a payoff $U_{i}(s)-\varepsilon$ at $s$.

c. $U$ possesses the complementary discontinuity property everywhere if, whenever $(s, u)$ is in the closure of the graph of its vector payoff function and $U_{i}(s) \leq u_{i}$ for every player $i$, then $U_{i}(s)=u_{i}$ for every player $i$.

Corollary 2 If the utility function possesses the complementary discontinuity property everywhere and is payoff secure everywhere then a pure strategy Nash equilibrium exists whenever an acceptable test sequence exists.

Proof. If the utility function is cdp and ps everywhere then it is cdp and ps for any acceptable test sequence. Thus the result follows directly from Theorem 1. QED.

Reny (1999, Theorem 3.1) provides an existence theorem with similar features. The difference between Corollary 1 and Reny's result is that this Corollary requires only the existence of an acceptable test sequence (a weaker condition than quasi concavity of the game) while Reny requires better-reply security (a weaker condition than cdp and ps).

Extending Theorem 1 and Corrolary 1 to allow for mixed strategy equilibrium is straight forward, requiring only the extension of definitions to the new setting. Of course the advantage of considering mixed strategies in this setting is that, applying Nash's theorem, every game with finite strategy spaces possesses a mixed strategy Nash equilibrium. Thus any test sequence $\tau$, where $\tau(n)$ is finite for all $n$ possesses an acceptable subsequence in mixed strategies.

Let $(M(S), \Psi)$ denote the mixed strategy extension of the limit game 
$G=(S, U)$, and $(M(\tau(n)), \Psi)$ denote the mixed strategy extension of $G^{n}=(\tau(n), U)$, where

$M(X)=\mathrm{X}_{i \in I} M\left(X_{i}\right)$ and $M\left(X_{i}\right)$ is the set of probability measures defined on the Borel subsets of $X_{i}$, and

$$
\Psi(\sigma)=\mathrm{X}_{i \in I} \Psi_{i}(\sigma) \text { where } \Psi_{i}: M(S) \rightarrow \mathbf{R} \text { such that } \Psi_{i}(\sigma)=
$$
$\int U_{i}(s) d \sigma$.

For every mixed strategy $\sigma(n) \in M(\tau(n))$ there exists an obvious extension $\sigma^{n} \in M(S)$, thus the any sequence of strategies $(\sigma(n))_{n \in \mathbf{N}}$ can be written as a sequence $\left(\sigma^{n}\right)_{n \in \mathbf{N}}$. As, by assumption, $S$ is compact and $U$ is bounded and Borel measurable, the set $M(S)$ paired with the Prohorov metric, $\rho$, is a compact metric space and $\Psi_{i}$ is bounded.

It follows directly that Theorem 1 can be applied to the mixed strategy specification $(M(S), \Psi)$ where the definitions of the complementary discontinuity property and payoff security are applied to the new 'mixed' strategy space $M(S)$ and 'expected' utility function $\Psi$. In practice some care is required in making the transition to the new specifications. Simon shows that even if the complementary discontinuity holds universally for the game $(S, U)$ it may fail in the mixed strategy extension $(M(S), \Psi)$ (Simon, footnote 7, p596). Similarly, the proof of the necessity condition also requires careful interpretation - as it is now the support of the equilibrium mixed strategies which must be available along test sequence.

Reny (Corollary 5.2) shows that any game where the expected utility function possesses the complementary and discontinuity property generally possesses a mixed strategy Nash equilibrium, and demonstrates that this result generalizes previous mixed strategy equilibrium results of Nash (1950), Glicksberg (1952), Mas-Colell (1984), Dasgupta and Maskin (1986), Robson (1994) and Simon (1987). Corollary 2 provides an alternative proof for this result.

Corollary 3 (Reny Corollary 5.2) The game (S,U) possesses a mixed 
strategy Nash equilibrium if the expected utility function $\Psi$ possesses the complementary discontinuity property and is payoff secure for all sequences $\left(\sigma^{n}\right)_{n \in \mathbf{N}}$.

Proof. As every finite test sequence is acceptable and generates a convergent sequence of Nash strategies, and the expected utility function possess the c.d. property and is payoff secure along every sequence, Theorem 1 must be satisfied. QED.

In the following section we further demonstrate the power of Theorem 1 by developing existence results for pure strategy and monotone equilibria in First-Price Auctions.

\section{Application: High Bid Auctions}

There are numerous examples of First Price or High Bid auctions which possess pure strategy Nash equilibria when the strategy is space is finite but where existence of a Nash equilibrium fails for continuous strategy spaces. ${ }^{5}$ The failure of existence with continuous strategy spaces is due to the possibility of ties, at winning bids, between bidders who value winning the auction differently. Jackson et al. (2001) show that there always exists a tie breaking rule which will support equilibrium in the continuous strategy game, and that this tie-breaking rule can be implemented with the addition of a 'cheap talk' phase to the auction game. However, two major caveats are identified for the generality and applicability of these results. First, they are considering equilibrium solutions in mixed strategies rather than pure strategies. Second, the tie-breaking rule in equilibrium will generally depend on the distribution of types of the bidders involved - information a real auctioneer is unlikely to possess. Thus, they note that

\footnotetext{
${ }^{5}$ Athey (2001), Jackson et al. (2001), Maskin \& Riley (2000), Reny \& Zamir (2003).
} 
"It seems an important challenge to identify circumstances in which uniform action rules - not depending on such information - suffice to guarantee existence of equilibrium." (Jackson et al. 2001, footnote 4, p4)

Maskin \& Riley (2000) address this problem directly and propose a two stage auction where, in the first stage, bidders participate in a standard 'High Bid' auction. If, however, two or more players submit the same highest bid then the tie is broken by a second round 'Vickery' auction amongst only the high bid players. Maskin \& Riley proceed to identify conditions which ensure that a monotonic pure strategy equilibrium exists for the two stage auction with continuous strategies (Proposition 2, p.444). As they demonstrate with an example (Example 3 , p.444), these conditions are more restrictive than required for the existence of a monotonic pure strategy equilibrium in the case of finite strategy spaces.

In this section we propose an alternative second round 'tie breaking' auction, specifically a cheap-talk high bid auction where bidders submit costless messages drawn from an ordered and finite message space. We show that, when the set of bidders and types are finite, this second round auction will support a pure strategy equilibrium under any conditions sufficient for equilibrium in finite strategy spaces (in fact the main result is more general than this). Furthermore equilibrium properties such as monotonicity that exist in the finite auctions will survive in the continuous strategy equilibrium. Thus for finite type spaces this result is a direct generalisation of Maskin \& Riley and, for example, allows us to directly extend any existence and monotonicity results generated in finite strategy spaces to continuous strategy spaces. ${ }^{6}$

To prove these assertions requires the introduction of further nota-

\footnotetext{
${ }^{6}$ Extending the results to continuous type space is objective of present research.
} 
tion. Consider a 'standard' finite type high bid auction, $A=\left(B ; u_{i} ; T_{i}\right)_{i \in I}$, where :

i. There is a finite set $(I)$ of potential bidders for a single, indivisible, product.

ii. Each player $i \in I$ has a type $t_{i} \in T_{i}$, where $T_{i}$ is a finite set, which is known to the player but unobserved other players. ${ }^{7}$ Let $f\left(t_{1}, \ldots, t_{I}\right)$ denote the probability of $\left(t_{1}, \ldots, t_{I}\right) \in \mathbf{X}_{i \in I} T_{i}$, and define the conditional probability

$$
g_{i}\left(t_{i} \mid t_{-i}\right)=\frac{f\left(t_{i}, t_{-i}\right)}{\sum_{t_{-i}^{\prime}} f\left(t_{i}, t_{-i}^{\prime}\right)}
$$

Assume further that, for all $i \in I$ and all $t_{i} \in T_{i}$, there exists $t_{-i}^{\prime} \in T_{-i}$ such that $f\left(t_{i}, t_{-i}^{\prime}\right)>0$, ensuring that $g_{i}\left(t_{i} \mid t_{-i}\right)$ is always well defined.

iii. Each player selects whether or not to participate in the auction, a bid $b_{i} \in B=[\underline{b}, \bar{b}] \subset \mathbf{R}$ and receives a payoff $u_{i}\left(b_{i}, t_{i}, \mathbf{t}_{-i}\right)$ if awarded the product auctioned, and zero otherwise. ${ }^{8},{ }^{9}$ Assume further that all payoff functions are bounded and are continuous and non-increasing with respect to the players bid, $b_{i}$.

iv. A strategy for each agent consists of a vector of type dependent bids which can be written $s_{i}=\left(b_{i}(t)\right)_{t \in T_{i}} \in \mathbf{X}_{t \in T_{i}} B=S_{i}$, where $S_{i}$ is a compact metric space.

The standard high bid auction awards the product to the participating bidder submitting the highest 'bid', or randomly amongst those

\footnotetext{
${ }^{7}$ Standard abuse of notation allows us to write $i \in I=\{1, \ldots, I\}$. For notational ease we restrict the set of types, $T$, and the space of bids, $B$, to be the same for all bidders - but the results can easily be extended to allow the type and strategy spaces to be type dependent.

${ }^{8}$ Following standard notation, let $x_{-i}$ denote the vector $x$ without the $i$ th component.

${ }^{9}$ The bounds on the set of bids could be justified by assuming either that the buyer and seller are wealth constrained, or by assuming that there exists a minimum (reservation) bid the seller will accept and a bid so high as to guarantee negative utility if successful. For simplicity we assume that the nonparticipation decision is represented by the bid $b_{i}=\varnothing$, and that the product auctioned will only ever be assigned to a participating bidder.
} 
bidders submitting the highest bid if more than one does so. Notice that the payoff functions from winning remain quite general, and may differ between bidders. It is NOT necessary that all winning bidders 'pay' their bid in full - thus this general specification allows the auctioneer to 'favour' one player over another when awarding the commodity.

We wish to augment the standard high-bid auction by introducing a second round 'cheap talk' high bid auction. Specifically we introduce a finite and ordered message space, $\Theta=\{0, \ldots, M\}$. Bidders who tie for the highest bid in the first round submit a costless message, $\theta_{i} \in \Theta$, and the auction winner is then selected randomly from amongst those who have submitted the highest message. We denote this 'cheap talk' message augmented auction by $\widehat{A}=\left((B, \Theta) ; u_{i} ; T_{i}\right)_{i \in I}$, and the set of Nash equilibrium in this auction $N(\widehat{A})$. As before, we will consider a sequence of approximations of the bid space $B^{n}$ converging to $B$, so $\lim _{n \rightarrow \infty} d^{H}\left(B^{n}, B\right)=0$, and the corresponding sequence of auctions $A^{n}=\left(\left(B^{n} ; u_{i} ; T_{i}\right)_{i \in I}\right.$.

The main result is now stated in Theorem 2, with the proof contained in the Appendix.

Theorem 4 For any acceptable sequence of high-bid auctions $A^{n}=$ $\left(\left(B^{n} ; u_{i} ; T_{i}\right)_{i \in I}\right.$ converging to $A=\left(B ; u_{i} ; T_{i}\right)_{i \in I}$, any $s^{*} \in N^{*}=$ $\lim _{n \rightarrow \infty} N\left(A^{n}\right)$, and any 'cheap talk' message augmented auction $\widehat{A}=$ $\left((B, \Theta) ; u_{i} ; T_{i}\right)_{i \in I}$ where $\# \Theta=M+1>T=\Sigma_{i \in I} T_{i}$;

there exist an equilibrium $\widehat{s^{*}} \in N(\widehat{A})$ such that $s^{*}$ and $\widehat{s^{*}}$ assign the same 'first round' bid to all players. That is, for any $i \in I$ and $t_{i} \in T_{i}$, if $s_{i}^{*}\left(t_{i}\right)=b \in B$ then $\widehat{s_{i}^{*}}\left(t_{i}\right)=\left(b, \theta_{i}\left(t_{i}\right)\right)$.

Furthermore, if there exists a sequence $s^{n} \in N\left(A^{n}\right)$ such that $\lim _{n \rightarrow \infty} s^{n}=s^{*}$ and $s^{n}$ is (strictly) monotonic for all $n$ sufficiently large, then so to is the equilibrium $\widehat{s^{*}} \in N(\widehat{A})$.

Once again, the application of Theorem 2 requires the existence of 
an acceptable test sequence to be demonstrated. The Theorem can be interpreted as saying that for any auction with finite types where an acceptable sequence can be shown to exist, imposing a 'cheap talk' high bid message auction as the tie breaking rule will ensure an equilibrium exists in the continuous auction. Notice that the theorem does not require, but certainly allows, the test sequence to be finite. Maskin \&

Riley (2000), for example, present three examples of first price auctions where Nash equilibria are shown to exist for any finite space of bids, but fail to exist when continuous bids are allowed. It follows directly from Theorem 2 that all three will necessarily have equilibria when the cheap talk high bid message tie breaking rule is imposed - which they show is not the case for the Vickery auction tie breaking rule they proposed. ${ }^{10}$

\section{Conclusion.}

In this paper we develop necessary sufficient conditions for the existence of Pure and Mixed Strategy Nash equilibrium in games with discontinuous payoffs and continuous strategy spaces. The approach is a direct generalization of the existence results developed by Dasgupta \& Maskin (1986) and Simon (1989), and the conditions identified differ from the sufficient conditions developed by Reny (1999). These differences are explained, and the result used to provide alternative proofs to some general existence results first presented by Reny. The main theorem (Theorem 1) identifies properties for the limit of a sequence of solutions to approximating games to be an equilibrium in the limit game. The properties identified are reasonably transparent and are particularly appropriate for testing computer generated solutions to specific games, where finite approximations of continuous strategy spaces are a technical necessity. In an application the main theorem is applied to

\footnotetext{
${ }^{10}$ Specifically, their Example 3 (p.444) does not possess an equilibrium with the Vickery tie-breaking rule.
} 
First-Price Auctions, where a specific 'cheap talk' tie breaking rule is proposed - with ties in the highest bid being broken by a second round, costless, High-Bid message auction. It is shown that implementing this tie breaking rule will ensure existence and (strict) monotonicity of Nash equilibria in continuous First-Price auctions whenever these properties exist in finite First-Price auctions. 


\section{References}

[1] Athey, S. (2001) 'Single crossing properties and the existence of pure strategy equilibria in games of incomplete information.' Econometrica, 69, 861-889.

[2] Baye, M.R., Tian, G. and Zhou, J. (1993) 'Characterizations of the existence of equilibria in games with discontinuous and nonquasiconcave payoffs.' Review of Economic Studies, 60, 935-948.

[3] Billingsley, P. (1968) Convergence of Probability Measures. Wiley.

[4] Billingsley, P. (1986) Probability and Measure (2nd ed.). Wiley.

[5] Dasgupta, P. and Maskin, E. (1986a) 'The existence of equilibrium in discontinuous economic games, I: Theory.' Review of Economic Studies, 53, 1-27.

[6] Dasgupta, P. and Maskin, E. (1986b) 'The existence of equilibrium in discontinuous economic games, II: Applications.' Review of Economic Studies, 53, 27-42

[7] Debreu, G. (1952) 'A social equilibrium existence theorem.' Proceedings of the National Academy of Sciences, 38, 886-893.

[8] Fudenberg, D. and Levine, D. (1986) 'Limit games and limit equilibria.' Journal of Economic Theory, 38, 261-279.

[9] Gatti, J.R.J. (1996) Multi-commodity search: Three essays. Ph.D. Thesis, University of Cambridge.

[10] Hildenbrand, W. (1974) Core and Equilibria of a Large Economy. Princeton University Press.

[11] Jackson, M.O., Simon, L.K., Swinkels, J.M., and Zame, W.R. (2001) 'Communication and equilibrium in discontinuous games of incomplete information.' Mimeo. 
[12] Nash, J. (1951) 'Non-cooperative games.', Annals of Mathematics, 54, 266-295.

[13] Reny, P.J. (2001) 'On the existence of pure and mixed strategy Nash equilibria in discontinuous games.' Econometrica, 67, 10291056 .

[14] Reny, P.J. and Zamir, S. (2004) 'On the existence of pure strategy monotone equilibria in asymmetric first-price auctions.' Econometrica, 72, 1105-1125.

[15] Simon, L.K. (1987) 'Games with discontinuous payoffs.' Review of Economic Studies, 54, 569-97.

[16] Simon, L.K. and Zame, W.R. (1990) 'Discontinuous games and endogenous sharing rules.' Econometrica, 58, 861-872.

[17] Sion, M. and Wolfe, P. (1957) 'On a game without a value.' Contributions to the Theory of Games, III. Annals of Mathematical Studies, 39, 299-306.

[18] Tian, G. (1992) 'Existence of equilibrium in abstract economies with discontinuous payoffs and non-compact choice spaces.' Journal of Mathematical Economics, 21, 379-388. 


\section{Appendix}

Proof of Theorem 2.

By definition, for every $s^{*} \in N^{*}=\lim _{n \rightarrow \infty} N\left(A^{n}\right)$ there exists a convergent subsequence $s^{n} \in N\left(A^{n}\right)$ such that $\lim _{n \rightarrow \infty} s^{n}=s^{*}$. The proof proceeds by showing that for every such subsequence $s^{n}$ it is possible to construct a sequence of (costly) message augmented auctions $\left(\widehat{A^{n}}\right)$ which converges to the costless message augmented auction $\widehat{A}$, and an associated sequence of Nash equilibria $\widehat{s^{n}} \in N\left(\widehat{A^{n}}\right)$ such that $\lim _{n \rightarrow \infty} \widehat{s^{n}}=\widehat{s^{*}} \in N(\widehat{A})$ and that the Nash equilibria $\widehat{s}^{*}$ and $s^{*}$ are related in the manner described in the Theorem.

We show (Lemma 3) that for any sequence $s^{n} \in N\left(A^{n}\right)$ there exists a sequence of finite high price auctions $\overline{A^{n}}$ such that $s^{n} \in N\left(\overline{A^{n}}\right)$ and $\lim _{n \rightarrow \infty} d^{H}\left(\overline{A^{n}}, A\right)=0$. We obtain conditions (Lemma 1) that enable a finite high bid auction to be represented by a strategically equivalent finite message-augmented auction. We then (Lemma 2) apply Theorem 1 to identify conditions which ensure that the limit of a sequence of equilibria of the finite message-augmented auctions is an equilibrium of the limiting message-augmented auction. We then show by construction (Lemma 3) that the limit of any sequence of Nash equilibria in high bid auctions can also be obtained as the first round bid component of the limit of a sequence of Nash equilibria in finite message-augmented auctions that satisfy the conditions identified in Lemma 2. Thus, as required, the limit of this sequence constitutes an equilibrium of the continuous-bid message augmented auction. Finally, we show that messages are costless in the limiting auction, and that the monotonicity property survives in the limit.

Some initial notation is required.

Let $X$ be a finite and ordered set. Without loss of generality, let $X=\left\{x_{1}, x_{2}, \ldots, x_{\# X}\right\}$ where $x_{1}<x_{2}<\ldots<x_{\# X}$. 
For any $x \in X$ let $R(x ; X): X \rightarrow \mathbf{N}$ denote the relative ranking of $x$ within $X$ and let $R^{-1}(i ; X): \mathbf{N} \rightarrow X$ be the inverse mapping. Thus $R\left(x_{i} ; X\right)=i$ and $R^{-1}(i ; X)=x_{i}$.

Define a mapping $\Phi: X \times Z_{+} \rightarrow X$ such that for any $x \in X$ and any non-negative integer $z \in Z_{+}, \Phi(x, z)=\{y \in X \mid R(y, X)=$ $\min \{R(x, X)+z, \# X\}$. Thus $\Phi(x, z)$ gives the element of $X$ which is ranked $z$ higher than $x$ or, if no such element exists, the largest element in the set $X$.

Lemma 5 finite first price auction $A^{n}=\left(\left(B^{n} ; u_{i} ; T_{i}\right)_{i \in I}\right.$ can be represented by a strategically equivalent finite 'message-augmented' high bid auction $\widehat{A^{n}}=\left(\left(\widehat{B^{n}}, \Theta\right) ; v_{i} ; T_{i}\right)_{i \in I}$, where $\Theta=\{0,1, \ldots, M\}$ and $v_{i}\left((b, \theta), t_{i}, t_{-i}\right)=u_{i}\left(\Phi(b, \theta), t_{i}, t_{-i}\right)$, if there exists $\widehat{B^{n}} \subseteq B^{n}$ such that the mapping $\Phi: \widehat{B^{n}} \times \Theta \rightarrow B^{n}$ is one-to-one and onto.

Furthermore, if auctions $A^{n}$ and $\widehat{A^{n}}$ are strategically equivalent, then for any $s^{n} \in N\left(A^{n}\right)$ there exists $\widehat{s}^{n} \in N\left(\widehat{A^{n}}\right)$ if and only if for all $i \in I$ and $t_{i} \in T_{i}, s_{i}^{n}\left(t_{i}\right)=\Phi\left(\widehat{s}_{i}^{n}\left(t_{i}\right)\right)$.

\section{Proof:}

If the mapping $\Phi: \widehat{B^{n}} \times \Theta \rightarrow B^{n}$ is 1-1 and onto then the two spaces map into each other uniquely and, given the utility function, the two auctions are identical providing they determine the winner identically. This requires that for all $\theta, \theta^{\prime} \in \Theta, \Phi(x, \theta)<\Phi\left(y, \theta^{\prime}\right)$ if $x<y$, and that $\Phi(x, \theta)<\Phi\left(x, \theta^{\prime}\right)$ if and only if $\theta<\theta^{\prime}$. These properties follow directly from the requirement that $\Phi: \widehat{B^{n}} \times \Theta \rightarrow B^{n}$ is 1-1 and onto - and so $\Phi(x, M)<\Phi(y, 1)$ if and only if $x<y \cdot Q E D$.

It may be worth noting that, as defined, the messages are not costless when the bid space is finite. However, as the distance between consecutive bids converges to zero, so too does the cost of any message. As in Theorem 1, we now consider a sequence of finite bid spaces $\left(B^{n}\right)$ 
converging to the continuous bid space $(B)$, so $\lim _{n \rightarrow \infty} B^{n}=B$, and the corresponding sequence of finite-bid message augmented auctions $\widehat{A^{n}}=\left(\left(\widehat{B^{n}}, \Theta\right) ; v_{i} ; T_{i}\right)_{i \in I}$ converging to the continuous bid message augmented auction $\widehat{A}=\left((B, \Theta) ; v_{i} ; T_{i}\right)_{i \in I}$. We will call this sequence of auctions acceptable if, for all $n$, there exists a Nash equilibrium $\widehat{s^{n}} \in N\left(\widehat{A^{n}}\right)$. Without loss of generality we can consider only convergent subsequences, for which $\lim _{n \rightarrow \infty} \widehat{s^{n}}=\widehat{s^{*}}$.

Lemma 6 If, for the message augmented auction $\widehat{A}$, there exists an acceptable sequence of finite message augmented auctions and a sequence of strategies $\left(\widehat{s^{n}}\right)_{n \in \mathbf{N}}$ where for all $n, \widehat{s}^{n} \in N\left(\widehat{A^{n}}\right)$, and $\lim _{n \rightarrow \infty} \widehat{s^{n}}=\widehat{s^{*}}=\left(b_{i}^{*}\left(t_{i}\right), \theta_{i}^{*}\left(t_{i}\right)\right)_{i \in I}$, such that

a. for all $i \in I, t_{i} \in T_{i}, \theta_{i}^{*}\left(t_{i}\right)<M$, and

$b$. if there exists $i, j \in I$ such that $b_{i}^{*}\left(t_{i}\right)=b_{j}^{*}\left(t_{j}\right)$ then $b_{i}^{n}\left(t_{i}\right)=b_{j}^{n}\left(t_{j}\right)$ for all $n$

$$
\text { then } \widehat{s^{*}} \in N(\widehat{A}) .
$$

\section{Proof:}

The proof is a direct application of Theorem 1. First we show that the conditions identified ensure that the payoff function possesses the complementary discontinuity property along the sequence, and then that the payoff function is also payoff secure along the sequence.

A players expected payoff in the auction can be written as

$$
V_{i}\left((b, \theta), \widehat{\mathbf{s}_{-i}} \mid t_{i}\right)=\sum_{l \geq 0}\left(\sum_{\mathbf{t}_{-i} \in P_{i}^{l}\left((b, \theta), \widehat{\mathrm{s}_{-i}}\right)}\left(\frac{1}{l+1}\right) v_{i}\left((b, \theta), t_{i}, \mathbf{t}_{-i}\right) f\left(\mathbf{t}_{-i} \mid t_{i}\right)\right) \quad(E q n A)
$$

where

$P_{i}^{l}\left((b, \theta), \widehat{\mathbf{s}_{-i}}\right)=\left\{\mathbf{t}_{-i} \in T_{-i} \mid\right.$ for all $j \neq i,\left(b_{j}\left(t_{j}\right), \theta_{j}\left(t_{j}\right)\right) \leq(b, \theta)$

and

$$
\left.\#\left\{j \in I \backslash i \mid\left(b_{j}\left(t_{j}\right), \theta_{j}\left(t_{j}\right)\right)=(b, \theta)\right\}=l\right\}
$$


denotes the subset of types where, given the strategies played by other players $\left(\mathbf{s}_{-i}\right)$, player $i$ making bid $b$ with message $\theta$ will tie for the highest bid with $l$ other players.

a. Complementary Discontinuity.

We show that, along the sequence, the payoff function for all players is continuous, and so the complementary discontinuity property is satisfied trivially.

By assumption, $v_{i}\left((b, \theta), t_{i}, \mathbf{t}_{-i}\right)=u_{i}\left(\Phi(b, \theta), t_{i}, \mathbf{t}_{-i}\right)$ is continuous in it's first term, so Eqn A shows that any discontinuity in $V_{i}\left((b, \theta), \widehat{\mathbf{s}_{-i}} \mid t_{i}\right)$ is due to a discontinuity in one or more of the partitions of the type space $P_{i}^{l}\left(b, \widehat{\mathbf{s}_{-i}}\right)$. More formally, along the sequence $\left(\widehat{s^{n}}\right)_{n \in \mathbf{N}}$,

$\lim _{n \rightarrow \infty} V_{i}\left(\left(b_{i}^{n}\left(t_{i}\right), \theta_{i}^{n}\left(t_{i}\right)\right), \widehat{\mathbf{s}_{-i}^{n}} \mid t_{i}\right)=V_{i}\left(\left(b_{i}^{*}\left(t_{i}\right), \theta_{i}^{*}\left(t_{i}\right)\right), \widehat{\mathbf{s}_{-i}^{*}} \mid t_{i}\right)$

if for all $l \geq 0, \lim _{n \rightarrow \infty} P_{i}^{l}\left(\left(b_{i}^{n}\left(t_{i}\right), \theta_{i}^{n}\left(t_{i}\right)\right), \widehat{\mathbf{s}_{-i}^{n}}\right)=P_{i}^{l}\left(\left(b_{i}^{*}\left(t_{i}\right), \theta_{i}^{*}\left(t_{i}\right)\right), \widehat{\mathbf{s}_{-i}^{*}}\right)$.

As $P_{i}^{l}\left(\left(b_{i}^{n}\left(t_{i}\right), \theta_{i}^{n}\left(t_{i}\right)\right), \widehat{\mathbf{s}}_{-i}^{n}\right)$ is a subset of a finite space the limit of this sequence is well defined and there exists a subsequence such that $P_{i}^{l}\left(\left(b_{i}^{n}\left(t_{i}\right), \theta_{i}^{n}\left(t_{i}\right)\right), \widehat{\mathbf{s}}_{-i}^{n}\right)=P \subseteq T_{-i}$ for all $n$. Discontinuity of this sequence in the limit requires that $P_{i}^{l}\left(\left(b_{i}^{*}\left(t_{i}\right), \theta_{i}^{*}\left(t_{i}\right)\right), \widehat{\mathbf{s}}_{-i}^{*}\right) \neq P$.

As the message space is finite we know that there exists a subsequence such that, for all $i \in I$ and $t_{i} \in T_{i}, \theta_{i}^{n}\left(t_{i}\right)=\theta_{i}^{*}\left(t_{i}\right)$. Condition (b) ensures that $b_{i}^{*}\left(t_{i}\right)=b_{j}^{*}\left(t_{j}\right)$ if and only if $b_{i}^{n}\left(t_{i}\right)=b_{j}^{n}\left(t_{j}\right)$ for all $n$. Thus $\mathbf{t}_{-i} \in P_{i}^{l}\left(\left(b_{i}^{n}\left(t_{i}\right), \theta_{i}^{n}\left(t_{i}\right)\right), \widehat{\mathbf{s}}_{-i}^{n}\right)=P_{i}^{l}\left(\left(b_{i}^{n}\left(t_{i}\right), \theta_{i}^{*}\left(t_{i}\right)\right), \widehat{\mathbf{s}}_{-i}\right)$ if and only if $\mathbf{t}_{-i} \in P_{i}^{l}\left(\left(b_{i}^{*}\left(t_{i}\right), \theta_{i}^{*}\left(t_{i}\right)\right), \widehat{\mathbf{s}_{-i}^{*}}\right)$, and so $P_{i}^{l}\left(\left(b_{i}^{*}\left(t_{i}\right), \theta_{i}^{*}\left(t_{i}\right)\right), \widehat{\mathbf{s}_{-i}^{*}}\right)=P$ as required.

b. Payoff security.

Let $Z_{t_{i}}\left(\widehat{\mathbf{s}_{-i}}\right)=\max _{(b, \theta) \in B \times \Theta} V_{i}\left((b, \theta), \widehat{\mathbf{s}_{-i}} \mid t_{i}\right)$ denote the best response value function for agent $i$ of type $t_{i}$,

and let $Z_{t_{i}}^{n}\left(\widehat{\mathbf{s}_{-i}^{n}}\right)=\max _{\left(b^{n}, \theta\right) \in B^{n} \times \Theta} V_{i}\left(\left(b^{n}, \theta\right), \widehat{\mathbf{s}}_{-i}^{n} \mid t_{i}\right)$. We show that the conditions in Lemma 2 ensure that for all $i \in I$ and $t_{i} \in T_{i}$ along the sequence $\left(\widehat{s^{n}}\right)_{n \in \mathbf{N}}$, and all $\epsilon>0, \lim _{n \rightarrow \infty} Z_{t_{i}}^{n}\left(\widehat{\mathbf{s}_{-i}^{n}}\right)>Z_{t_{i}}\left(\widehat{\mathbf{s}_{-i}^{*}}\right)-\epsilon$.

For any $X \subseteq B$ and $s_{-i} \in \mathbf{X}_{t_{j}, j \neq i}(X, \Theta)$; let 
$\beta\left(s_{-i}\right)=\left\{x \in X \mid\right.$ there exists $j \in I \backslash i$ and $t_{j} \in T_{j}$ such that $\left.s_{j}\left(t_{j}\right)=\left(x, \theta_{j}\left(t_{j}\right)\right)\right\}$ denote the set of bids that may be realised by players other than $i$, given $s_{-i}$.

Suppose that $z \in B$ and $m \in \Theta$ are best responses for agent $i \in I$ of type $t_{i} \in T_{i}$, so $V_{i}\left((z, m), \widehat{\mathbf{s}_{-i}^{*}} \mid t_{i}\right)=Z_{t_{i}}\left(\widehat{\mathbf{s}_{-i}^{*}}\right)$. We consider separately the case where $z \in \beta\left(\widehat{s_{-i}^{*}}\right)$ and $z \notin \beta\left(\widehat{s}_{-i}^{*}\right)$.

i) If $z \in \beta\left(\widehat{s_{-i}^{*}}\right)$, then there exists a sequence $z^{n} \in \beta\left(\widehat{s_{-i}^{n}}\right)$ where $\lim _{n \rightarrow \infty} z^{n}=z$ and there exists $\bar{n}<\infty$ such that for all $n>\bar{n}$

$$
\begin{aligned}
& Z_{t_{i}}^{n}\left(\widehat{\mathbf{s}_{-i}^{n}}\right) \geq V_{i}\left(\left(z^{n}, m\right), \widehat{\mathbf{s}_{-i}^{n}} \mid t_{i}\right)>V_{i}\left(\left(z^{*}, m\right), \widehat{\mathbf{s}_{-i}^{n}} \mid t_{i}\right)-\frac{\epsilon}{2}= \\
& Z_{t_{i}}\left(\mathbf{s}_{-i}^{*}\right)-\frac{\epsilon}{2},
\end{aligned}
$$

thus $\lim _{n \rightarrow \infty} Z_{t_{i}}^{n}\left(\widehat{\mathbf{s}_{-i}^{n}}\right)>Z_{t_{i}}\left(\widehat{\mathbf{s}_{-i}^{*}}\right)-\epsilon$, as required.

ii) If $z \notin \beta\left(\widehat{s_{-i}^{*}}\right)$, then consider the largest observable bid less than $z$, specifically consider $y=\left\{\max x \in \beta\left(\widehat{s}_{-i}^{*}\right)\right.$ such that $\left.x<z\right\}$. By construction no other player of any type is playing a strategy with a bid in the range $(y, z]$, and by assumption no player of any type is playing $(y, M)$. Thus for all $b \in(y, z], \theta \in \Theta$ and $l \geq 0$, the subsets $P_{i}^{l}\left((z, m), \widehat{\mathbf{s}_{-i}^{*}}\right)=P_{i}^{l}\left((b, \theta), \widehat{\mathbf{s}_{-i}^{*}}\right)=P_{i}^{l}\left((y, M), \widehat{\mathbf{s}_{-i}^{*}}\right)$ and $P_{i}^{l}\left((z, m), \widehat{\mathbf{s}_{-i}^{*}}\right)=\varnothing$ if $l>0$. By assumption we have that $v_{i}\left((b, \theta), t_{i}, \mathbf{t}_{-i}\right)$ is continuous and non-increasing in $b$ and $m$ thus, from Eqn. A we have that $V_{i}\left((y, M), \widehat{\mathbf{s}_{-i}^{*}} \mid t_{i}\right) \geq V_{i}\left((z, m), \widehat{\mathbf{s}}_{-i}^{*} \mid t_{i}\right)$. As $V_{i}\left((z, m), \widehat{\mathbf{s}_{-i}^{*}} \mid t_{i}\right)=Z_{t_{i}}\left(\widehat{\mathbf{s}_{-i}^{*}}\right)$ we have that $V_{i}\left((y, M), \widehat{\mathbf{s}_{-i}^{*}} \mid t_{i}\right)=$ $Z_{t_{i}}\left(\widehat{\mathbf{s}_{-i}^{*}}\right)=V_{i}\left((z, m), \widehat{\mathbf{s}_{-i}^{*}} \mid t_{i}\right)$. As $y \in \beta\left(\widehat{s_{-i}^{*}}\right)$ we can now apply part (i) to show that $\lim _{n \rightarrow \infty} Z_{t_{i}}^{n}\left(\widehat{\mathbf{s}_{-i}^{n}}\right)>Z_{t_{i}}\left(\widehat{\mathbf{s}}_{-i}^{*}\right)-\epsilon$, as required. This completes the proof of Lemma 2.

Lemma 7 For any acceptable sequence of high-bid auctions $A^{n}=$ $\left(\left(B^{n} ; u_{i} ; T_{i}\right)_{i \in I}\right.$ converging to $A=\left(B ; u_{i} ; T_{i}\right)_{i \in I}$ and any $s^{*} \in N^{*}=$ $\lim _{n \rightarrow \infty} N\left(A^{n}\right)$, we can construct a sequence of strategically equivalent message augmented auctions $\widehat{A^{n}}=\left(\left(\widehat{B^{n}}, \Theta\right) ; v_{i} ; T_{i}\right)_{i \in I}$ with $\#\{\Theta\}>T$ that converges to $\widehat{A}=\left((B, \Theta) ; v_{i} ; T_{i}\right)_{i \in I}$ and a possesses sequence of Nash equilibrium strategies $\left(\widehat{s^{n}}\right)_{n \in \mathbf{N}}$ converging to $\widehat{s}^{*}$ which satisfy the 
conditions of Lemma 2 (thus $\widehat{s^{*}} \in N(\widehat{A})$ ) and where $s^{*}$ and $\widehat{s^{*}}$ assign the same 'first round' bid to all players.

\section{Proof:}

The proof makes use of the fact that one can systematically remove unused strategies from players without disrupting Nash equilibrium. More formally, consider a game $G=(S, U)$ and a Nash equilibrium $s \in N(G)$, if $s \in S^{\prime} \subset S$ and $G^{\prime}=\left(S^{\prime}, U\right)$ then $s \in N\left(G^{\prime}\right)$. The proof proceeds in three steps.

\section{Step 1}

Consider the sequence of finite auctions $\left(A^{n}\right)_{n \in \mathbf{N}}$ converging to $A$ and any sequence of Nash equilibrium strategies $\left(s^{n}\right)_{n \in \mathbf{N}}$ converging to $s$. For every $b \in \beta(s)$ there exists a non-empty subset of players whose equilibrium strategies converge to $b$, that is for which $\lim _{n \rightarrow \infty} s_{i}^{n}\left(t_{i}\right)=b$. Consider the set $B^{n}(b)=\left\{x \in B^{n} \mid\right.$ there exists $\left(i, t_{i}\right)$ such that $\lim _{n \rightarrow \infty} s_{i}^{n}\left(t_{i}\right)=b$ and $\left.s_{i}^{n}\left(t_{i}\right)=x\right\}$. As the sets of agents and types are finite there are at most $T=\sum_{i \in I} T_{i}$ elements in $B^{n}(b)$. Let $\underline{b^{n}}$ (resp. $\left.\overline{b^{n}}\right)$ denote the min $(\max )$ values of $B^{n}(b)$, and consider the set $\left.\overline{B_{b}^{n}}=\left(B^{n} \backslash \underline{b^{n}}, \overline{b^{n}}\right]\right) \cup B^{n}(b)$, which removes all the 'unused' values of $B^{n}$ within the range $\left[\underline{b^{n}}, \overline{b^{n}}\right]$.

Consider a message space $\Theta=\{0,1, \ldots, M\}$ and the mapping $\Phi$ : $\overline{B_{b}^{n}} \times \Theta \rightarrow \overline{B_{b}^{n}}$. Providing $M \geq T$, for all $x \in B^{n}(b)$ there exists $\theta<T$ such that $\Phi(\underline{b}, \theta)=x$, and for all $\theta \geq T$ have $\Phi(\underline{b}, \theta)>\bar{b}$.

Notice that for any pair of bids $b, b^{\prime} \in \beta(s), b \neq b^{\prime}$ if and only if $\left[\underline{b^{n}}, \overline{b^{n}}\right] \cap\left[\underline{b^{n \prime}}, \overline{b^{n \prime}}\right]=\varnothing$ for all $n$ sufficiently large. (If not, there exists a sequence of bids that converges to both $b$ and $b^{\prime}$, thus $\left.b \neq b^{\prime}\right)$.

We can now repeat this deletion procedure for every $b \in \beta(s)$, to obtain

$$
\overline{B^{n}}=B^{n} \backslash\left\{\bigcup_{b \in \beta(s)}\left[\underline{b^{n}}, \overline{b^{n}}\right]\right\} \cup\left\{\bigcup_{b \in \beta(s)} B^{n}(b)\right\} .
$$

By definition $s^{n} \in N\left(A^{n}\right)$ along the sequence $\left(s^{n}\right)_{n \in \mathbf{N}}$ converging to 
s. By construction $s^{n} \in \overline{B^{n}} \subseteq B^{n}$ and $\lim _{n \rightarrow \infty} \overline{B^{n}}=B$. Thus, for the high bid auction $\overline{A^{n}}=\left(\overline{B^{n}} ; u_{i} ; T_{i}\right)_{i \in I}$ we have that $s^{n} \in N\left(\overline{A^{n}}\right)$ and the sequence of auctions $\overline{A^{n}}$ is acceptable and converges to $A$.

Step 2.

Consider now a set $\widehat{B^{n}} \subseteq \overline{B^{n}}$ such that

i. $R^{-1}\left(1 ; \widehat{B^{n}}\right)=R^{-1}\left(1 ; \overline{B^{n}}\right)$, so the lowest element of both sets is the same

ii. for every $b \in \beta(s), \underline{b^{n}} \in \widehat{B^{n}}$, so the lowest element of every set of sequences converging to $b$ is an element

iii. For any $x, y \in \widehat{B^{n}}$ such that $R\left(y ; \widehat{B^{n}}\right)=R\left(x ; \widehat{B^{n}}\right)+1$, we have that $M<R\left(y ; \overline{B^{n}}\right)-R\left(x ; \overline{B^{n}}\right)<2 M+1$ and, furthermore, $R\left(y ; \overline{B^{n}}\right)-R\left(x ; \overline{B^{n}}\right)=M+1$ if $y \neq \underline{b^{n}}$ for some $b \in \beta(s)$. Thus $\widehat{B^{n}}$ takes every $(M+1)$ th element of $\overline{B^{n}}$, with the proviso that the lowest element defined in (ii) are elements and no two elements have a rank difference of less than $M+1$ in $\overline{B^{n}}$.

Now, consider the space $\widetilde{B^{n}} \subseteq \overline{B^{n}}$ defined by the set $\widehat{B^{n}}$ and the mapping $\Phi: \overline{B^{n}} \times \Theta \rightarrow \overline{B^{n}}$, so that $x \in \widetilde{B^{n}}$ if and only if there exist $y \in \widehat{B^{n}}$ and $\theta \in \Theta$ such that $\Phi(y, \theta)=x$. By construction,

for all $n, s^{n} \in \widetilde{B^{n}}$ thus $s^{n} \in \widetilde{A^{n}}=\left(\widetilde{B^{n}} ; u_{i} ; T_{i}\right)_{i \in I}$

$d^{H}\left(\widetilde{B^{n}}, B\right) \leq(2 M+1) d^{H}\left(B^{n}, B\right)$, so the sequence $\widetilde{B^{n}}$ converges to $B$, and the sequence $\widetilde{A^{n}}$ converges to $A$ and is acceptable

the mapping $\Phi$ is one to one and onto

\section{Step 3}

We can now apply Lemma 1, to show that the sequence of message augmented auctions $\widehat{A^{n}}=\left(\left(\widehat{B^{n}}, \Theta\right) ; v_{i} ; T_{i}\right)_{i \in I}$ are strategically equivalent to the acceptable sequence of auctions $\widetilde{A^{n}}=\left(\widetilde{B^{n}} ; u_{i} ; T_{i}\right)_{i \in I}$.

By the construction of $\widehat{B^{n}}$, for all $b \in \beta(s)$ and all $\left(b_{i}^{n}\left(t_{i}\right), \theta_{i}^{n}\left(t_{i}\right)\right)$ converging to $(b, \theta), b_{i}^{n}\left(t_{i}\right)=\underline{b^{n}}$ and $\theta_{i}^{n}\left(t_{i}\right)=\theta<T \leq M$. 
Thus we can apply Lemma 2, to show the limit of the sequence $\widehat{s^{n}}$ is a Nash equilibrium of the limit game $\widehat{A}=\left((B, \Theta) ; v_{i} ; T_{i}\right)_{i \in I}$. This completes the proof of Lemma 3.

Finally to consider monotonicity we need to define an ordering $(O)$ over the space $B \times M$. Specifically we shall select the lexicographic ordering, s.t. $\left(b_{1}, m_{1}\right)>_{O}\left(b_{2}, m_{2}\right)$ if and only if $b_{1}>b_{2}$ or $b_{1}=b_{2}$ and $m_{1}>m_{2}$. Clear if the strategies along the sequence $s^{n}$ are (strictly) monotonic then the corresponding strategies in the message augmented auctions must also be monotonic and either assign higher bids, or higher message. As neither bids nor messages converge in the limit - the limiting strategies must also preserve monotonicity. This completes the proof of Theorem 2 . 